\title{
Remote Quantum-Information Concentration: Reversal of Ancilla-Free Phase-Covariant Telecloning
}

\author{
Xinwen Wang ${ }^{1}$, Shiqing Tang ${ }^{2}$ \\ ${ }^{1}$ Key Laboratory of Low-Dimensional Quantum Structures and Quantum Control of Ministry of Education, \\ Department of Physics, Hunan Normal University, Changsha, China \\ ${ }^{2}$ Department of Physics and Electronic Information Science, Hengyang Normal University, Hengyang, China \\ Email: wangxinwen@hynu.cn
}

Received December 16, 2012; revised January 18, 2013; accepted January 26, 2013

\begin{abstract}
Telecloning and its reverse process, referred to as remote quantum-information concentration (RQIC), have been attracting considerable interest because of their potential applications in quantum-information processing. The previous RQIC protocols were focused on the reverse process of the optimal universal telecloning. We here study the reverse process of ancilla-free phase-covariant telecloning (AFPCT). It is shown that the quantum information originally distributed into two spatially separated qubits from a single qubit via the optimal AFPCT procedure can be remotely concentrated back to a single qubit with a certain probability by using an asymmetric $\mathrm{W}$ state as the quantum channel.
\end{abstract}

Keywords: Remote Quantum-Information Concentration; Phase-Covariant Telecloning; W State

\section{Introduction}

It is well known that an unknown quantum state can not be perfectly copied because of the quantum no-cloning theorem $[1,2]$. Since the seminal work of Bužek and Hillery [3], however, approximate (the fidelities of clones are less than one) or probabilistic quantum cloning has been attracting much attention (see [4] for a review and outlook), due to the fact that it has wide potential applications in quantum-information science as well as could help us understand quantum mechanics itself more well (see, e.g. [5-9]). The quantum cloning process can be in fact regarded as the distribution of quantum information from an initial system to final ones. For realizing the remote information distribution with quantum cloning, $\mathrm{Mu}-$ rao et al. $[10,11]$ introduced the concept of telecloning, which is the combination of quantum cloning and quantum teleportation [12]. Telecloning functions as simultaneously distributing the copies of an unknown quantum state to spatially separated sites, i.e., realizing nonlocal quantum cloning, via a previously shared multipartite entangled state. Telecloning has been widely studied and many idiographic schemes have been proposed [13-18]. As the reverse process of telecloning, remote quantuminformation concentration (RQIC) was first introduced by Murao and Vedral [19]. They demonstrated that the quantum information originally distributed into three spatially separated qubits from a single qubit by the optimal universal telecloning procedure [10] can be remotely concentrated back to a single qubit via a four-qubit unlock- able bound entangled state [20] assisted by local operations and classical communication (LOCC). Telecloning and RQIC processes could be regarded as, respectively, remote quantum-information depositing and withdrawing processes, or remote quantum-information encoding and decoding processes, which are expected to find useful applications in network-based quantum-information processing [19]. A scheme for implementing the reversal of the optimal $1 \rightarrow 2$ universal telecloning via a four-qubit Greenberger-Horne-Zeilinger (GHZ) state [21] has also been proposed [22]. Recently, RQIC has been generalized to many-particle and high-dimensional systems [2325].

All the previous RQIC protocols were focused on the reverse process of the optimal universal telecloning. In this paper, we investigate the reverse process of ancillafree phase-covariant telecloning. Particularly, we present a scheme for implementing $2 \rightarrow 1 \mathrm{RQIC}$, i.e., the reversal of the optimal $1 \rightarrow 2$ ancilla-free phase-covariant telecloning (AFPCT) for qubits. The entangled channel used in our RQIC protocol is the same as that used in the optimal $1 \rightarrow 2$ AFPCT [14], a tripartite asymmetric W state. This result indicates that both the telecloning and RQIC tasks can be realized by utilizing the same entangled channel. However, it will be shown that the $\mathrm{W}$ state can only implement the RQIC probabilistically, in contrast to fact that it can implement the optimal $1 \rightarrow 2$ AFPCT deterministically.

Before describing our RQIC protocol, we briefly sum- 
marize the forward process, the optimal $1 \rightarrow 2$ AFPCT [14]. Such an AFPCT scheme aims at simultaneously distributing two optimal clones of a phase-covariant state

$$
|\phi\rangle_{A}=\frac{1}{\sqrt{2}}\left(|0\rangle+\mathrm{e}^{\mathrm{i} \delta}|1\rangle\right)_{A}
$$

from a distributor (Alice) to two spatially separated receivers (Bob and Charlie) with only LOCC. Here $\{|0\rangle,|1\rangle\}$ represents the computational basis for a qubit and $\delta$ is an unknown phase. The entangled channel is a three-qubit asymmetric W state [26]

$$
|W\rangle_{A^{\prime} B^{\prime} C^{\prime}}=\frac{1}{\sqrt{2}}|100\rangle_{A^{\prime} B^{\prime} C^{\prime}}+\frac{1}{2}(|010\rangle+|001\rangle)_{A^{\prime} B^{\prime} C^{\prime}}
$$

where qubit $A^{\prime}$ is the input port of the distributor Alice, and qubits $B^{\prime}$ and $C^{\prime}$ are the output ports for the clones held, respectively, by the two receivers Bob and Charlie (throughout the paper, if necessary, the subscripts of the kets or the operators denote the qubit index). Here we have adopted the notation $|x y z\rangle=|x\rangle|y\rangle|z\rangle$. The telecloning can be accomplished by the following simple procedure: 1) Alice performs a Bell-state measurement on qubits $A$ and $A^{\prime}$, obtaining one of four Bell states, and informs Bob and Charlie of the outcome; 2) depending on Alice's measurement outcome, Bob and Charlie perform appropriate local unitary operations on the qubits they hold, obtaining the clones of $|\phi\rangle$ with the optimal fidelity $F=1 / 2+1 / 2 \sqrt{2}$ $[27,28]$. The collective output state of qubits $B^{\prime}$ and $C^{\prime}$ is the entangled state

$$
|\psi\rangle_{B^{\prime} C^{\prime}}=\frac{1}{\sqrt{2}}|00\rangle_{B^{\prime} C^{\prime}}+\frac{1}{2} e^{i \delta}(|01\rangle+|10\rangle)_{B^{\prime} C^{\prime}}
$$

We now describe our RQIC protocol for implementing the reversal of the aforementioned telecloning. After telecloning operations, the initial single-qubit $(A)$ quantum information is remotely distributed into two spatially separated qubits ( $B^{\prime}$ and $C^{\prime}$ ), represented by the collective quantum state in Equation (3). The ownership of particles $B^{\prime}$ and $C^{\prime}$ is the same as before; i.e., they are still held by Bob and Charlie, respectively. The RQIC is aim to concentrate the information initially distributed in two-qubit cloning state of Equation (3) back to a remote particle $D$ (held by Diana) with only LOCC: $|\psi\rangle_{B^{\prime} C^{\prime}} \rightarrow|\phi\rangle_{D}$. We also consider employing the following tripartite asymmetric $\mathrm{W}$ entangled state as the quantum channel:

$$
|W\rangle_{B C D}=\frac{1}{\sqrt{2}}|001\rangle_{B C D}+\frac{1}{2}(|010\rangle+|100\rangle)_{B C D}
$$

where particles $B, C$, and $D$ belong to Bob, Charlie, and Diana, respectively. The RQIC task can be accomplished by three steps: (S1) Bob and Charlie perform Bell-state measurements on their own particles, respectively; (S2) Each of them tells Diana the measurement outcome by sending 2 bits of classical information; (S3) Diana performs a conditional local operation on particle $\mathrm{D}$.
The detailed explanation for the above procedure is as follows. According to Equations (3) and (4), the state of the whole system (qubits $\left\{B^{\prime}, C^{\prime}, B, C, D\right\}$ ) is given by

$$
\begin{aligned}
& |\Omega\rangle_{B^{\prime} C^{\prime} B C D}=|\psi\rangle_{B^{\prime} C^{\prime}} \otimes|W\rangle_{B C D} \\
& =\frac{1}{2}|00001\rangle_{B^{\prime} C^{\prime} B C D}+\frac{1}{2 \sqrt{2}}(|00010\rangle+|00100\rangle)_{B^{\prime} C^{\prime} B C D} \\
& +\frac{1}{4} \mathrm{e}^{\mathrm{i} \delta}(|01010\rangle+|01100\rangle+|10010\rangle+|10100\rangle)_{B^{\prime} C^{\prime} B C D} \\
& +\frac{1}{2 \sqrt{2}} \mathrm{e}^{\mathrm{i} \delta}(|01001\rangle+|10001\rangle)_{B^{\prime} C^{\prime} B C D}
\end{aligned}
$$

With the Bell-basis states

$$
\begin{aligned}
& \left|\Phi^{ \pm}\right\rangle=\frac{1}{\sqrt{2}}(|00\rangle \pm|11\rangle) \\
& \left|\Psi^{ \pm}\right\rangle=\frac{1}{\sqrt{2}}(|01\rangle \pm|10\rangle)
\end{aligned}
$$

The total quantum state $|\Omega\rangle_{B^{\prime} C^{\prime} B C D}$ can be expanded as

$$
\begin{aligned}
& |\Omega\rangle_{B^{\prime} C^{\prime} B C D}=\frac{1}{2 \sqrt{2}}\left|\Phi^{+}\right\rangle_{B^{\prime} B}\left|\Phi^{+}\right\rangle_{C^{\prime} C} \frac{1}{\sqrt{2}}\left(|1\rangle+\mathrm{e}^{\mathrm{i} \delta}|0\rangle\right)_{D} \\
& +\frac{1}{2 \sqrt{2}}\left|\Phi^{-}\right\rangle_{B^{\prime} B}\left|\Phi^{-}\right\rangle_{C^{\prime} C} \frac{1}{\sqrt{2}}\left(|1\rangle-\mathrm{e}^{\mathrm{i} \delta}|0\rangle\right)_{D} \\
& +\frac{1}{4}\left(\left|\Phi^{+}\right\rangle_{B^{\prime} B}+\left|\Phi^{-}\right\rangle_{B^{\prime} B}\right)\left|\Psi^{+}\right\rangle_{C^{\prime} C} \frac{1}{\sqrt{2}}\left(|0\rangle+\mathrm{e}^{\mathrm{i} \delta}|1\rangle\right)_{D} \\
& +\frac{1}{4}\left|\Psi^{+}\right\rangle_{B^{\prime} B}\left(\left|\Phi^{+}\right\rangle_{C^{\prime} C}+\left|\Phi^{-}\right\rangle_{C^{\prime} C}\right) \frac{1}{\sqrt{2}}\left(|0\rangle+\mathrm{e}^{\mathrm{i} \delta}|1\rangle\right)_{D} \\
& +\frac{1}{4}\left(\left|\Phi^{+}\right\rangle_{B^{\prime} B}+\left|\Phi^{-}\right\rangle_{B^{\prime} B}\right)\left|\Psi^{-}\right\rangle_{C^{\prime} C} \frac{1}{\sqrt{2}}\left(|0\rangle-\mathrm{e}^{\mathrm{i} \delta}|1\rangle\right)_{D} \\
& +\frac{1}{4}\left|\Psi^{-}\right\rangle_{B^{\prime} B}\left(\left|\Phi^{+}\right\rangle_{C^{\prime} C}+\left|\Phi^{-}\right\rangle_{C^{\prime} C}\right) \frac{1}{\sqrt{2}}\left(|0\rangle-\mathrm{e}^{\mathrm{i} \delta}|1\rangle\right)_{D} \\
& +\frac{1}{4}\left(\left|\Phi^{+}\right\rangle_{B^{\prime} B}\left|\Phi^{-}\right\rangle_{C^{\prime} C}+\left|\Phi^{-}\right\rangle_{B^{\prime} B}\left|\Phi^{+}\right\rangle_{C^{\prime} C}\right)|1\rangle_{D} \\
& +\frac{1}{4}\left(\left|\Psi^{+}\right\rangle_{B^{\prime} B}\left|\Psi^{+}\right\rangle_{C^{\prime} C}+\left|\Psi^{-}\right\rangle_{B^{\prime} B}\left|\Psi^{-}\right\rangle_{C^{\prime} C}\right) \mathrm{e}^{\mathrm{i} \delta}|0\rangle_{D}
\end{aligned}
$$

It can be seen from Equation (7) that when the combinational Bell-state measurement outcome is in the set

$$
\begin{array}{r}
S_{1}=\left\{\left|\Phi^{+}\right\rangle_{B^{\prime} B}\left|\Psi^{+}\right\rangle_{C^{\prime} C},\left|\Phi^{-}\right\rangle_{B^{\prime} B}\left|\Psi^{+}\right\rangle_{C^{\prime} C}\right. \\
\left.\left|\Psi^{+}\right\rangle_{B^{\prime} B}\left|\Phi^{+}\right\rangle_{C^{\prime} C},\left|\Psi^{+}\right\rangle_{B^{\prime} B}\left|\Phi^{-}\right\rangle_{C^{\prime} C}\right\}
\end{array}
$$

the particle $D$ will be projected in the state $|\phi\rangle$, meaning the success of RQIC. If the double Bell-state measurement outcome belongs to the following set $S_{2}$, the particle $D$ can also be prepared in the state $|\phi\rangle$, by suitable local unitary transformations (see Table 1):

$$
\begin{aligned}
S_{2}= & \left\{\left|\Phi^{+}\right\rangle_{B^{\prime} B}\left|\Phi^{+}\right\rangle_{C^{\prime} C},\left|\Phi^{-}\right\rangle_{B^{\prime} B}\left|\Phi^{-}\right\rangle_{C^{\prime} C}\right. \\
& \left|\Phi^{+}\right\rangle_{B^{\prime} B}\left|\Psi^{-}\right\rangle_{C^{\prime} C},\left|\Phi^{-}\right\rangle_{B^{\prime} B}\left|\Psi^{-}\right\rangle_{C^{\prime} C} \\
& \left.\left|\Psi^{-}\right\rangle_{B^{\prime} B}\left|\Phi^{+}\right\rangle_{C^{\prime} C},\left|\Psi^{-}\right\rangle_{B^{\prime} B}\left|\Phi^{-}\right\rangle_{C^{\prime} C}\right\}
\end{aligned}
$$


Table 1. The correspondence between the local unitary operations needing to be performed on qubit $D$ and the Bellstate measurement outcomes in the set $S_{2}$ on the two pairs of qubits $\left(B^{\prime}, B\right)$ and $\left(C^{\prime}, C\right) \cdot \sigma^{x, y, z}$ denote conventionally the Pauli operators and $I$ is the identity operator.

\begin{tabular}{cc}
\hline Measurement outcomes & Transformations \\
\hline$\left|\Phi^{+}\right\rangle_{B^{\prime} B}\left|\Phi^{+}\right\rangle_{C^{C} C}$ & $\sigma_{D}^{x}$ \\
$\left|\Phi^{-}\right\rangle_{B^{\prime} B}\left|\Phi^{-}\right\rangle_{C C}$ & $\mathrm{i} \sigma_{D}^{y}$ \\
$\left|\Phi^{+}\right\rangle_{B^{\prime} B}\left|\Psi^{-}\right\rangle_{C C}$ & \\
$\left|\Phi^{-}\right\rangle_{B^{\prime} B}\left|\Psi^{-}\right\rangle_{C^{C} C}$ & \\
$\left|\Psi^{-}\right\rangle_{B^{\prime} B}\left|\Phi^{+}\right\rangle_{C C}$ & $\sigma_{D}^{z}$ \\
$\left|\Psi^{-}\right\rangle_{B^{\prime} B}\left|\Phi^{-}\right\rangle_{C^{C} C}$ & \\
\hline
\end{tabular}

As to the physical realization of the above RQIC scheme, one mainly needs to consider three points: 1) preparation of the entangled channel, i.e., the asymmetric $\mathrm{W}$ state of Equation (4);2) realization of the optimal $1 \rightarrow$ 2 ancilla-free telecloning (or cloning) of the phase-covariant state $|\phi\rangle$ in Equation (1);3) implementation of the Bell-state measurement. Recently, some schemes for generating the asymmetric W state of Equation (4) have been presented in different physical systems [29-33]. Several physical schemes for realizing the optimal $1 \rightarrow 2$ ancilla-free phase-covariant telecloning or cloning have also been proposed $[33,34]$. Based on the current techniques $[35,36]$, these schemes are experimentally achievable. The Bell-state measurement can be well realized for both atomic and photonic qubits $[37,38]$. Very recently, we have also proposed a near deterministic scheme [39] for realizing nondestructively the photonic Bell-state (or GHZ-state) measurement with the two-photon parity gate based on cross-Kerr nonlinearity (see Ref. [40] and the references therein). All these achievements may contribute to our RQIC scheme in physical realization.

In conclusion, we have presented a new RQIC scheme for implementing the reversal of the optimal $1 \rightarrow 2$ AFPCT, in contrast to the previous RQIC schemes for implementing the reversal of the optimal universal telecloning. The entangled channel used in our protocol is an experimentally realizable three-qubit $\mathrm{W}$ state. Such a RQIC protocol is expected to find useful applications in the field of modern multiparty quantum-information processing. In addition, the idea may open another perspective for the applications of the $\mathrm{W}$ states.

There are two open questions that deserve further investigating in the future. 1) Whether the reverse process of the optimal $1 \rightarrow 2$ AFPCT could be implemented deterministically? 2) Whether the reverse process of $1 \rightarrow n$ AFPCT could be realized?

\section{Acknowledgments}

This work was supported by the National Natural Science Foundation of China (Grant No. 11004050), the Key Project of Chinese Ministry of Education (Grant No. 211119), and the China Postdoctoral Science Foundation funded project (Grant No. 2012M511729).

\section{REFERENCES}

[1] W. K. Wootters and W. H. Zurek, "A Single Quantum Cannot Be Cloned," Nature, Vol. 299, No. 5886, 1982, pp. 802-803. doi:10.1038/299802a0

[2] D. Dieks, "Communication by EPR Devices," Physics Lettters A, Vol. 92, No. 6, 1982, pp. 271-272. doi:10.1016/0375-9601(82)90084-6

[3] V. Bužek and M. Hillery, "Quantum Copying, beyond the No-Cloning Theorem," Physical Review A, Vol. 54, No. 3, 1996, pp. 1844-1852. doi:10.1103/PhysRevA.54.1844

[4] V. Scarani, et al., "Quantum Cloning," Reviews of Modern Physics, Vol. 77, No. 4, 2005, pp. 1225-1256. doi:10.1103/RevModPhys.77.1225

[5] E. F. Galvao and L. Hardy, "Cloning and Quantum Computation," Physical Review A, Vol. 62, No. 2, 2000, Article ID: 022301. doi:10.1103/PhysRevA.62.022301

[6] M. Ricci, et al., "Separating the Classical and Quantum Information via Quantum Cloning," Physical Review Letters, Vol. 95, No. 9, 2005, Article ID: 090504. doi:10.1103/PhysRevLett.95.090504

[7] D. Bruß, J. Calsamiglia and N. Lutkenhaus, "Quantum Cloning and Distributed Measurements," Physical Review A, Vol. 63, No. 4, 2001, Article ID: 042308. doi:10.1103/PhysRevA.63.042308

[8] P. Sekatski, et al., "Cloning Entangled Photons to Scales One Can See," Physical Review A, Vol. 82, No. 5, 2010, Article ID: 053814. doi:10.1103/PhysRevA.82.053814

[9] N. Spagnolo, F. Sciarrino and F. De Martini, "Resilience to Decoherence of the Macroscopic Quantum Superpositions Generated by Universally Covariant Optimal Quantum Cloning," Physical Review A, Vol. 82, No. 3, 2010, Article ID: 032325. doi:10.1103/PhysRevA.82.032325

[10] M. Murao, et al., "Quantum Telecloning and Multiparticle Entanglement," Physical Review A, Vol. 59, No. 1, 1999, pp. 156-161. doi:10.1103/PhysRevA.59.156

[11] M. Murao, M. B. Plenio and V. Vedral, "Quantum-Information Distribution via Entanglement," Physical Review $A$, Vol. 61, No. 3, 2000, Article ID: 032311. doi:10.1103/PhysRevA.61.032311

[12] C. H. Bennett, et al., "Teleporting an Unknown Quantum State via Dual Classical and Einstein-Podolsky-Rosen Channels," Physical Review Letters, Vol. 70, No. 13, 1993, pp. 1895-1899. doi:10.1103/PhysRevLett.70.1895

[13] I. Ghiu, "Asymmetric Quantum Telecloning of $D$-Level Systems and Broadcasting of Entanglement to Different Locations Using the 'Many-To-Many' Communication Protocol," Physical Review A, Vol. 67, No. 1, 2003, Article ID: 012323. doi:10.1103/PhysRevA.67.012323 
[14] X. W. Wang and G. J. Yang, "Hybrid Economical Telecloning of Equatorial Qubits and Generation of Multipartite Entanglement," Physical Review A, Vol. 79, No. 6, 2009, Article ID: 062315.

doi:10.1103/PhysRevA.79.062315

[15] I. Ghiu and A. Karlsson, "Broadcasting of Entanglement at a Distance Using Linear Optics and Telecloning of Entanglement," Physical Review A, Vol. 72, No. 3, 2005, Article ID: 032331. doi:10.1103/PhysRevA.72.032331

[16] L. Chen and Y. X. Chen, "Asymmetric Quantum Telecloning of Multiqubit States," Quantum Information and Computation, Vol. 7, 2007, pp. 716-729.

[17] X. W. Wang and G. J. Yang, "Probabilistic Ancilla-Free Phase-Covariant Telecloning of Qudits with the Optimal Fidelity," Physical Review A, Vol. 79, No. 6, 2009, Article ID: 064306. doi:10.1103/PhysRevA.79.064306

[18] X. W. Wang, Y. H. Su and G. J. Yang, "One-to-Many Economical Phase-Covariant Cloning and Telecloning of Qudits," Chinese Physics Letters, Vol. 27, No. 10, 2010, Article ID: 100303. doi:10.1088/0256-307X/27/10/100303

[19] M. Murao and V. Vedral, "Remote Information Concentration Using a Bound Entangled State," Physical Review Letters, Vol. 86, No. 2, 2001, pp. 352-355. doi:10.1103/PhysRevLett.86.352

[20] J. A. Smolin, "Four-party Unlockable Bound Entangled State," Physical Review A, Vol. 63, No. 3, 2001, Article ID: 032306. doi:10.1103/PhysRevA.63.032306

[21] D. M. Greenberger, et al., "Bells Theorem without Inequalities," American Journal of Physics, Vol. 58, 1990, pp. 1131-1143. doi:10.1119/1.16243

[22] Y. F. Yu, J. Feng and M. S. Zhan, "Remote Information Concentration by a Greenberger-Horne-Zeilinger State and by a Bound Entangled State," Physical Review A, Vol. 68, No. 2, 2003, Article ID: 024303. doi:10.1103/PhysRevA.68.024303

[23] R. Augusiak and P. Horodecki, "Generalised Smolin states and Their Properties," Physical Review A, Vol. 73, No. 1, 2006, Article ID: 012318. doi:10.1103/PhysRevA.73.012318

[24] L. Y. Hsu, "Remote One-Qubit Information Concentration and Decoding of Operator Quantum Error-Correction Codes," Physical Review A, Vol. 76, No. 3, 2007, Article ID: 032311. doi:10.1103/PhysRevA.76.032311

[25] X. W. Wang, et al., "Remote Information Concentration and Multipartite Entanglement in Multilevel Systems," Physical Review A, Vol. 84, No. 4, 2011, Article ID: 042310. doi:10.1103/PhysRevA.84.042310

[26] W. D"ur, et al., "Three Qubits Can Be Entangled in Two Inequivalent Ways," Physical Review A, Vol. 62, No. 6, 2000, Article ID: 062314. doi:10.1103/PhysRevA.62.062314

[27] D. Bruß, et al., "Phase-Covariant Quantum Cloning," Physical Review A, Vol. 62, No. 1, 2000, Article ID: 012302. doi:10.1103/PhysRevA.62.012302
[28] G. M. D'Ariano and C. Macchiavello, "Optimal PhaseCovariant Cloning for Qubits and Qutrits," Physical Review A, Vol. 67, No. 4, 2003, Article ID: 042306. doi:10.1103/PhysRevA.67.042306

[29] X. W. Wang, et al., "Simple Schemes for Quantum Information Processing with W-Type Entanglement," $Q u$ antum Information Processing, Vol. 8, 2009, pp. 431-442. doi:10.1007/s11128-009-0119-8

[30] X. W. Wang, "Preparation and Manipulation of W-Class Entangled States: Applications to Quantum Information Processing," International Journal of Quantum Information, Vol. 7, No. 2, 2009, pp. 493-504. doi:10.1142/S0219749909004633

[31] Z. H. Peng, et al., "Scheme for Implementing Efficient Quantum Information Processing with Multiqubit WClass States in Cavity QED," Journal of Physics B, Vol. 41, 2008, Article ID: 065505. doi:10.1088/0953-4075/41/6/065505

[32] Y. H. Wang and H. S. Song, "Preparation of Partially Entangled W State and Deterministic Multi-controlled Teleportation," Optics Communications, Vol. 281, 2008, pp. 489-493. doi:10.1016/j.optcom.2007.09.057

[33] Q. Liu, W. H. Zhang and L. Yie, "Scheme to Implement Scheme $1 \rightarrow M$ Economical Phase-Covariant Telecloning via Cavity QED," Chinese Physics Letters, Vol. 25, No. 6, 2008, pp. 1947-1949. doi:10.1088/0256-307X/25/6/007

[34] W. H. Zhang, J. L. Dai and L. Yie, "Scheme to Implement Optimal Asymmetric Economical Phase-Covariant Quantum Cloning in Cavity QED," Communications in Theoretical Physics, Vol. 49, No. 6, 2008, pp. 1473-1476. doi:10.1088/0253-6102/49/6/27

[35] S. Gleyzes, et al., "Quantum Jumps of Light Recording the Birth and Death of a Photon in a Cavity," Nature, Vol. 446, No. 7133, 2007, pp. 297-300. doi: $10.1038 /$ nature 05589

[36] H. Mabuchi and A. C. Doherty, "Cavity Quantum Electrodynamics: Coherence in Context," Science, Vol. 298, No. 5597, 2002, pp. 1372-1377. doi:10.1126/science.1078446

[37] R. Reichle, et al., "Experimental Purification of TwoAtom Entanglement," Nature, Vol. 443, No. 7113, 2006, pp. 838- 841. doi:10.1038/nature05146

[38] S. Olmschenk, et al., "Quantum Teleportation between Distant Matter Qubits," Science, Vol. 323, No. 5913, 2009, pp. 486-489. doi:10.1126/science.1167209

[39] X. W. Wang, et al., "Nondestructive Greenberger-HorneZeilinger-State Analyzer," Quantum Information Processing, Vol. 12, No. 2, 2013, pp. 1065-1075. doi:10.1007/s11128-012-0453-0

[40] X. W. Wang, et al., "Photonic Two-Qubit Parity Gate with Tiny Cross-Kerr Nonlinearity," Physical Review A, Vol. 85, No. 5, 2012, Article ID: 052326. doi:10.1103/PhysRevA.85.052326 